\title{
Simultaneous Screening of 177 Drugs of Abuse in Urine Using Ultra-performance Liquid Chromatography with Tandem Mass Spectrometry in Drug-intoxicated Patients
}

\author{
Yong-Wha Lee \\ Department of Laboratory Medicine and Genetics, Soonchunhyang University Bucheon Hospital, Soonchunhyang University College of \\ Medicine, Bucheon, Korea
}

\begin{abstract}
Objective: The demand for rapid and broad clinical toxicology screening methods to identify drugs of abuse and medicinal drugs is increasing steadily. Liquid chromatography-tandem mass spectrometry (LC-TMS) is increasingly used to screen for drugs of abuse and to identify a wide range of drugs and metabolites in clinical samples. We revised a high-throughput and rapid ultra-performance (UP) LC-TMS method for simultaneous screening of 177 of the most prevalent medicinal drugs and drugs of abuse in urine and validated the quality of performance using system suitability mixture (SSM) and quality control (QC) materials. Methods: We assessed the limits of detection (LOD) using high concentrations of the test substances. The method was applied to 473 urine samples obtained from patients intoxicated with drugs who visited the emergency center.

Results: The retention time, peak area, and total ion chromatogram of the SSM and QC materials were within the acceptance criteria of the pre-defined acceptance interval. The LODs were $<62 \mathrm{ng} / \mathrm{ml}$ for 12 commonly encountered drugs. In total, 418 patients (88.4\%) tested positive for one or more medicinal drugs or drugs of abuse. Twenty-eight drugs were detected over ten times; the most commonly detected were zolpidem, ephedrine, paracetamol, and chlorpheniramine.

Conclusion: The UPLC-TMS method provided excellent performance for simultaneous screening of a large number of the drugs of abuse in urine samples. We conclude that this robust technique is useful for screening for a large number of drugs and for rapid screening of the most commonly encountered substances in emergency cases.
\end{abstract}

KEY WORDS: Drugs of abuse; Liquid chromatography; Tandem mass spectrometry; Toxicology.

\section{INTRODUCTION}

The demand for rapid and broad clinical toxicology screening methods to identify drugs of abuse and medicinal drugs is steadily increasing. This is primarily related to an increasing number of therapeutic drugs and drugs of abuse as well as samples submitted for analysis. Screening for substances is performed using immunoassays, gas chromatography mass spectrometry (GC-MS), liquid chromatography or liquid chromatography mass spectrometry (LC-MS). ${ }^{1-5)}$

Immunoassays for screening drugs of abuse are rapid; however, these methods are calibrated at cut-off levels

\footnotetext{
Received: September 25, 2013 / Revised: November 4, 2013

Accepted: November 7, 2013

Address for correspondence: Yong-Wha Lee, MD, PhD Department of Laboratory Medicine and Genetics, Soonchunhyang University Bucheon Hospital, Soonchunhyang University College of Medicine, 170 Jomaru-ro, Wonmi-gu, Bucheon 420-767, Korea Tel: +82-32-621-5943, Fax: +82-32-621-5944 E-mail: lywmd@schmc.ac.kr
}

that are higher than the detection limit to ensure reliability. Therefore, these methods lack sensitivity and specificity, leading to false-negative results and false-positive screening results. ${ }^{6}$ Automated high performance liquid chromatography system has been a useful complementary technique in the clinical toxicology laboratory. ${ }^{7)}$ Only GC-MS can detect a variety of drugs; however, this method involves a laborious and time-consuming chemical derivatization of nonvolatile and polar analytes. ${ }^{8,9)}$

LC-MS/MS has been increasingly used in clinical toxicology to identify a wide range of drugs and metabolites in clinical samples. ${ }^{10)}$ The major advantages of the LCMS/MS technique are ease of sample preparation, that no derivatization required, the short analysis time, and simultaneous screening of analytes with higher sensitivity and selectivity. ${ }^{11,12)}$ In screening of drugs of abuse, LC-MS/MS has demonstrated sufficient validity to replace previous screening methodologies. ${ }^{13)}$

Ultra-performance liquid chromatography-tandem mass

(c) This is an Open-Access article distributed under the terms of the Creative Commons Attribution Non-Commercial License (http://creativecommons.org/licenses/by-nc/3.0) which permits unrestricted non-commercial use, distribution, and reproduction in any medium, provided the original work is properly cited. 
spectrometry (UPLC-TMS) has been used for screening of drugs of abuse and therapeutic drugs in urine, blood, and hair as sample matrices. Urine contains both the drug itself and its metabolites, and in many cases the detection window is longer in urine than in blood. There is an increasing need to broaden the range of drugs that can be screened for simultaneously using a limited sample.

In this study, we revised a high-throughput, rapid and robust UPLC-TMS method that involves simple pretreatment for the simultaneous screening of a large number of drugs of abuse in urine. A total of 177 of the most prevalent medicinal drugs and drugs of abuse was included, and the method was successfully applied to 473 urine samples obtained from patients intoxicated with drugs who visited the emergency center.

\section{METHODS}

\section{Materials and Reagents}

Urine samples (stored at $4^{\circ} \mathrm{C}$ until tested) were collected from patients intoxicated with drugs who visited the emergency center in Soonchunhyang University Bucheon Hospital (Bucheon, Korea) between July 2011 and June 2013. All samples were anonymized and subjected to UPLC-TMS analysis within 3 days of collection.

All solvents were LC-MS grade. Methanol and acetonitrile were obtained from Duksan (Ansan, Korea) and formic acid and ammonium acetate were purchased from Sigma-Aldrich (St. Louis, MO, USA). The system suitability mixture (SSM; Sigma-Aldrich) including reserpine, doxepine, doxylamine, colchicine, caffeine, and imipramine was prepared for validation of quality.

SSM was reconstituted to $1 \mu \mathrm{g} / \mathrm{ml}$ of six compounds in $5 \mathrm{mM}$ ammonium formate ( $\mathrm{pH}$ 3.0). A 150- $\mu 1$ aliquot of urine was transferred to an individual $1.5-\mathrm{ml}$ tube. Then, $150 \mu 1$ of acetonitrile were added to precipitate the protein. The mixture was mixed using a vortex mixer for 1 min until the sample was thoroughly dissolved and centrifuged at 13,000 rpm for $5 \mathrm{~min}$. The supernatant was diluted fivefold using $400 \mu 1$ of LC-MS grade water. Sample aliquots were injected into the UPLC-TMS for analysis.

\section{UPLC-TMS}

UPLC was performed on a Waters Acquity ${ }^{\circledR}$ UPLC system (Waters Corp., Milford, MA, USA). The autosampler injected $10 \mu \mathrm{l}$ of extract into an Acquity ${ }^{\circledR}$ UPLC HSS C18 column $(2.1 \times 150 \mathrm{~mm}, 1.8 \mu \mathrm{m})$ maintained at $50^{\circ} \mathrm{C}$ in a column oven. LC separation of the drugs was performed using a gradient profile of mobile phase A and B solutions, consisting of $5 \mathrm{mM}$ ammonium formate ( $\mathrm{pH}$ 3.0) and acetonitrile with $0.1 \%$ formic acid $(\mathrm{v} / \mathrm{v})$, respectively. The flow rate was $400 \mu \mathrm{l} / \mathrm{min}$ and running time was $15 \mathrm{~min}$. The gradient program was $13 \% \mathrm{~B}$ increased to $95 \% \mathrm{~B}$ at $400 \mu \mathrm{l} / \mathrm{min}$ for $15 \mathrm{~min}$. The total instrumental analysis time was $17 \mathrm{~min}$, including column re-equilibration.

TMS was used to detect 177 drugs with a Waters Acquity ${ }^{\circledR}$ TQ-Detector (Table 1). At unit mass resolution, the mass analyzer had the following settings: cone voltage, 25-55 V; collision energy, 10-50 eV; source and desolvation temperatures, 150 and $400^{\circ} \mathrm{C}$, respectively; and desolvation gas flow, $800 \mathrm{~L} / \mathrm{h}$. The analysis was performed in single multiple reaction monitoring (MRM) mode for each compound and was electrospray ionization positive using the transactions: mass to charge ratio $(\mathrm{m} / \mathrm{z})$ (MRMs available upon request). Detection was performed using TargetLynx Manager in the Waters MassLynx 4.1 software (Waters Corp., Milford, MA, USA). A single MRM transition (two daughter ions) for each compound in addition to its expected retention time was obtained in the preconfigured TargetLynx method (available upon request). After processing, a list of compounds with peak areas above a pre-defined threshold was reviewed.

\section{Method Validation}

\section{Quality control}

The quality of the performance using $1 \mu \mathrm{g} / \mathrm{ml} \mathrm{SSM}$ was assessed using the following acceptance criteria: a pre-defined retention time acceptance interval of within $0.2 \mathrm{~min}$, peak area of $>200$ and total ion chromatogram of $>1.0 \times 10^{4}$ for six compounds in each batch. Additionally, quality control (QC) using two levels (negative and positive) of commercially available QC materials (Liquichek ${ }^{\mathrm{TM}}$ Qualitative Urine Toxicology Control; Bio-Rad, Hercules, CA, USA) was analyzed in each run; the QC material contains 12 commonly encountered substances.

\section{Limits of detection (LOD)}

LOD was evaluated using the positive control material, which comprised commercially available drug of abuse QC materials. LOD was determined by twofold serial dilution of the Liquichek ${ }^{\mathrm{TM}}$ material with the following minimum criteria: a pre-defined retention time acceptance interval of within $0.2 \mathrm{~min}$, peak area of $>100$ and total ion chromatogram of $>1.0 \times 10^{3}$ for each analyte. The LOD was defined as the lowest concentration that corresponded to the minimum criteria. 
Table 1. The list of 177 drugs detectable using ultra performance liquid chromatography with tandem mass spectrometry

\begin{tabular}{|c|c|c|c|c|c|}
\hline & Drugs detected & & Drugs detected & & Drugs detected \\
\hline 1 & 6-Monoacetylmorphine & 60 & EDDP & 119 & Nitrazepam \\
\hline 2 & 7 amino-clonazepam & 61 & Ephedrine & 120 & Norbuprenorphine \\
\hline 3 & 7 amino-flunitrazepam & 62 & Estazolam & 121 & Nordiazepam \\
\hline 4 & 7 amino-nitrazepam & 63 & Ethenzamide & 122 & Norketamine \\
\hline 5 & Acebutolol & 64 & Fenspiride & 123 & Nortriptyline \\
\hline 6 & Acepromazine & 65 & Fentanyl & 124 & Olanzapine \\
\hline 7 & Alimemazine & 66 & Flecainide & 125 & Oxazepam \\
\hline 8 & Alprazolam & 67 & Flumazenil & 126 & Oxcarbazepine \\
\hline 9 & Ambroxol & 68 & Flunitrazepam & 127 & Oxprenolol \\
\hline 10 & Amiodarone & 69 & Fluoxetine & 128 & Oxycodone \\
\hline 11 & Amisulpride & 70 & Fluphenazine & 129 & Paracetamol \\
\hline 12 & Amitriptyline & 71 & Flurazepam & 130 & Paroxetine \\
\hline 13 & Amoxapine & 72 & Fluvoxamine & 131 & Phencyclidine \\
\hline 14 & Amphetamine & 73 & Haloperidol & 132 & Perphenazine \\
\hline 15 & Atenolol & 74 & Heroin & 133 & Phenacetin \\
\hline 16 & Atropine & 75 & Hydrocodone & 134 & Pheniramine \\
\hline 17 & Benzoylecgonine & 76 & Hydromorphone & 135 & Phenylpropanolamine \\
\hline 18 & Betaxolol & 77 & Hydroquinidine & 136 & Phenytoin \\
\hline 19 & Bisoprolol & 78 & Hydroxy Alprazolam & 137 & Pindolol \\
\hline 20 & Bromazepam & 79 & Hydroxyzine & 138 & Piroxicam \\
\hline 21 & Brompheniramine & 80 & Imipramine & 139 & Prazepam \\
\hline 22 & Buflomedil & 81 & Indomethazin & 140 & Primidone \\
\hline 23 & Bupivacaine & 82 & Ketamine & 141 & Procaine \\
\hline 24 & Buprenorphine & 83 & Labetalol & 142 & Promethazine \\
\hline 25 & Bupropion & 84 & Lamotrigine & 143 & Propafenone \\
\hline 26 & Caffeine & 85 & Levomepromazine & 144 & Propoxyphene \\
\hline 27 & Carbamazepine & 86 & Lidocaine & 145 & Propranolol \\
\hline 28 & Celiprolol & 87 & Loprazolam & 146 & Pseudoephedrine \\
\hline 29 & Chlordiazepoxide & 88 & Lorazepam & 147 & Quetiapine \\
\hline 30 & Chloroquine & 89 & Lormetazepam & 148 & Quinidine \\
\hline 31 & Chlorpheniramine & 90 & Loxapine & 149 & Rantitidine \\
\hline 32 & Chlorpromazine & 91 & LSD & 150 & Reserpine \\
\hline 33 & Citalopram & 92 & Maprotiline & 151 & Rispéridone \\
\hline 34 & Clenbuterol & 93 & $\mathrm{MBDB}$ & 152 & Salbutamol \\
\hline 35 & Clobazam & 94 & MDA & 153 & Scopolamine \\
\hline 36 & Clomipramine & 95 & MDEA & 154 & Sertraline \\
\hline 37 & Clonazepam & 96 & MDMA & 155 & Sotalol \\
\hline 38 & Clonidine & 97 & Meloxicam & 156 & Strychnine \\
\hline 39 & Clotiazepam & 98 & Meperidine & 157 & Sulindac \\
\hline 40 & Cloxazolam & 99 & Meprobamate & 158 & Sulpiride \\
\hline 41 & Clozapine & 100 & Methadone & 159 & Temazepam \\
\hline 42 & Cocaine & 101 & Methamphetamine & 160 & Tetracaine \\
\hline 43 & Codeine & 102 & Methocarbamol & 161 & Tetrazepam \\
\hline 44 & Colchicine & 103 & Methyl Clonazepam & 162 & Theophylline \\
\hline 45 & Desalkyl flurazepam & 104 & Methylphenidate & 163 & Thioridazine \\
\hline 46 & Desipramine & 105 & Metoclopramide & 164 & Tianeptine \\
\hline 47 & Dextromethorphan & 106 & Metoprolol & 165 & Tiapride \\
\hline 48 & Dextromoramide & 107 & Mianserine & 166 & Tofisopam \\
\hline 49 & Diazepam & 108 & Midazolam & 167 & Topiramate \\
\hline 50 & Dihydrocodeine & 109 & Milnacipran & 168 & Tramadol \\
\hline 51 & Diltiazem & 110 & Mirtazapine & 169 & Trazadone \\
\hline 52 & Diphenydramine & 111 & Molsidomine & 170 & Triazolam \\
\hline 53 & Disopyramide & 112 & Morphine & 171 & Trifluoperazine \\
\hline 54 & Domperidone & 113 & Nadolol & 172 & Trimipramine \\
\hline 55 & Doxapram & 114 & Nalbuphine & 173 & Tripolidine \\
\hline 56 & Doxepine & 115 & Nalorphine & 174 & Venlafaxine \\
\hline 57 & Doxylamine & 116 & Naltrexone & 175 & Verapamil \\
\hline 58 & Droperidol & 117 & Naproxen & 176 & Zolpidem \\
\hline 59 & Ecgonine methyl ester & 118 & Nicotine & 177 & Zopiclone \\
\hline
\end{tabular}

EDDP, 2-ethylidene-1,5-dimethyl-3,3-diphenylpyrrolidine; LSD, lysergic acid diethylamide; MBDB, N-methyl-1,3-benzodioxolylbutanamine; MDA, 3,4-methylenedioxyamphetamine; MDEA, 3,4-methylenedioxy-N-ethylamphetamine; MDMA, 3,4-methylenedioxy-N-methylamphetamine. 


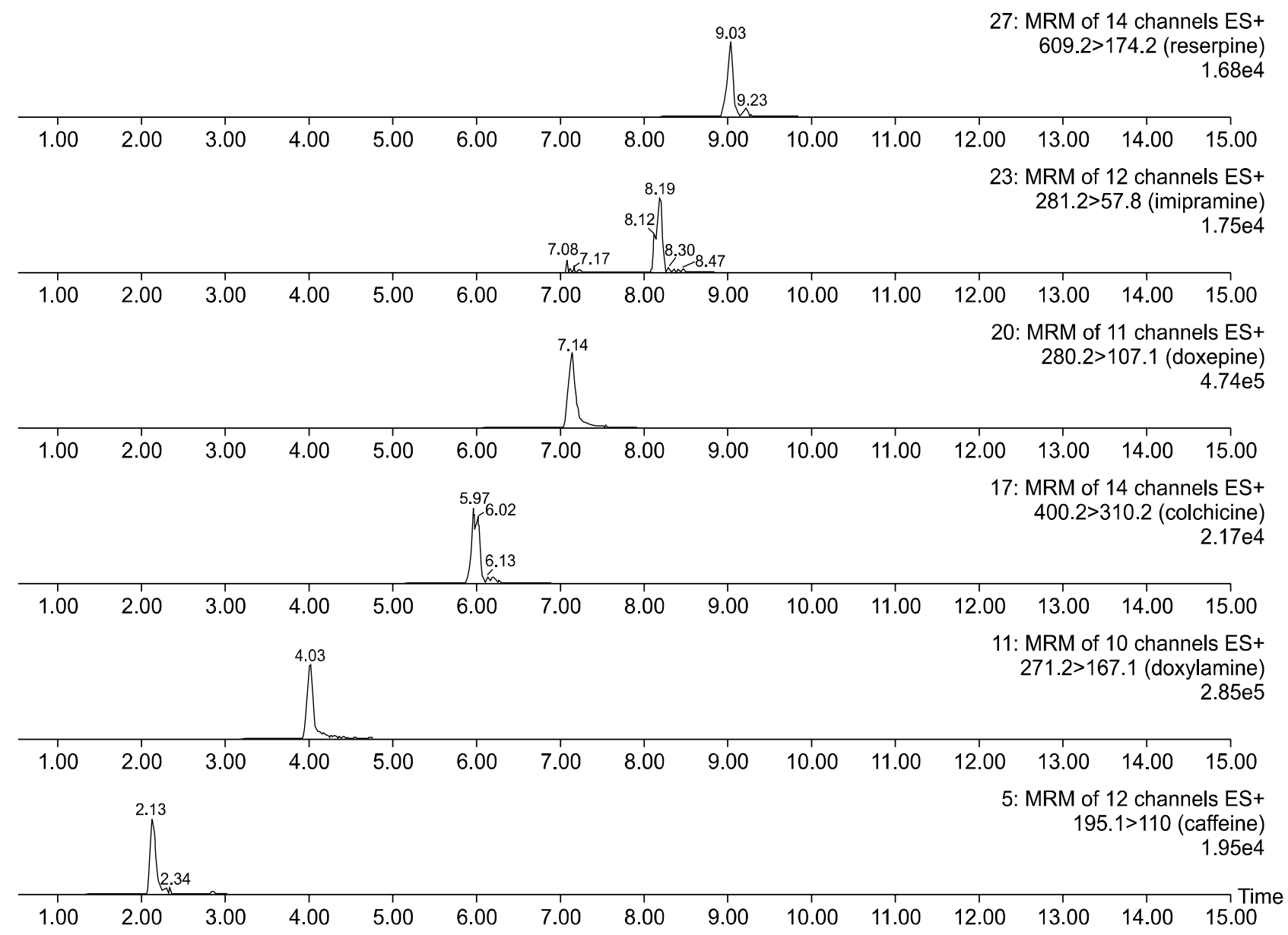

Fig. 1. Chromatograms of the system suitability mixture, which included caffeine, doxylamine, colchicine, doxepine, imipramine, and reserpine, as generated by ultra-performance liquid chromatography-tandem mass spectrometry.

ES+, electrospray ionization positive mode.

3. Distribution of drugs detected in 473 drug-intoxicated patients

Drug intoxication screening using the UPLC-TMS method was performed in 473 patients who were suspected of drug intoxication and who visited the emergency center from July 2011 to June 2013. The number of patients with a confirmed diagnosis of drug intoxication and the characteristics of the drugs detected were investigated. The study was approved by the institutional review board of Soonchunhyang University Bucheon Hospital (IRB 2013-10-030).

\section{RESULTS}

\section{SSM and Screened Drug UPLC-TMS Chromatograms}

Six isolated peaks were separated chromatographically. Excellent peak shapes were observed for SSM, including caffeine, doxylamine, colchicine, doxepine, imipramine, and reserpine (Fig. 1). The retention times were 2.13, $4.03,6.00,7.14,8.19$, and $9.03 \mathrm{~min}$, respectively.

The single MRM transition for each compound in addition to its expected retention time was provided in the preconfigured method for screening purposes. After processing, a list of compounds with peak areas above a pre-defined threshold was viewed (Fig. 2).

\section{UPLC-TMS Performance Validation}

The retention time, peak area, and total ion chromatogram of the SSM were within the acceptance criteria of the pre-defined acceptance interval. Additionally, the reproducibility tests using negative and positive QC materials showed results consistent with the target values for all analytes.

The LODs determined were $<62 \mathrm{ng} / \mathrm{ml}$ for 12 commonly encountered drugs from the QC material. The LODs of MDMA (3,4-methylenedioxy-N-methylamphe- 


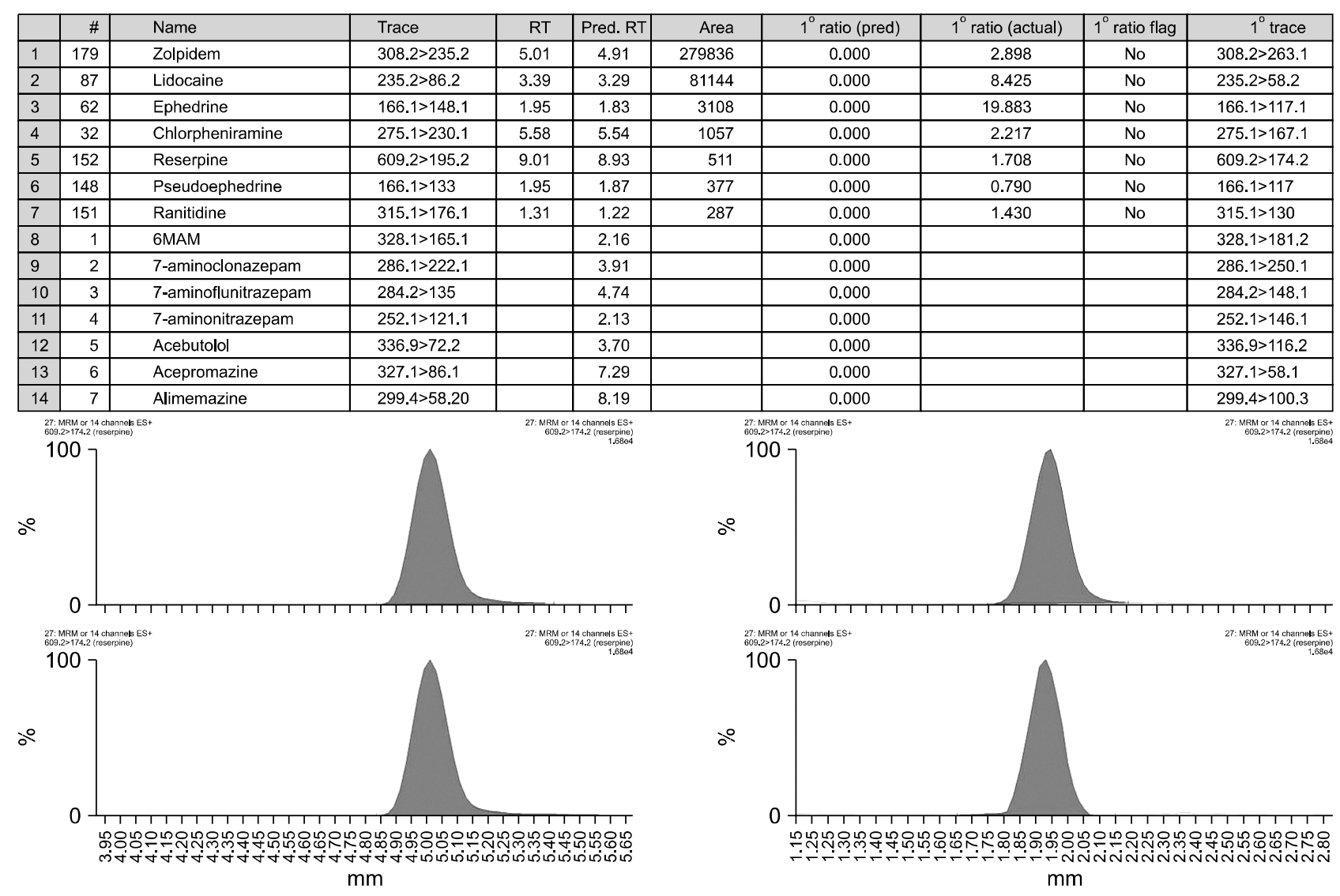

Fig. 2. Drugs with peak areas above a pre-defined (Pred.) threshold and chromatograms of the identified substances (i.e. zolpidem). RT, retention time.

Table 2. Limits of detection for the 12 commonly encountered drugs contained in the quality control materials

\begin{tabular}{|c|c|c|c|c|c|c|c|c|c|c|}
\hline \multirow[b]{2}{*}{ Drug } & \multirow{2}{*}{$\begin{array}{c}\text { Assigned } \\
\text { concentration } \\
(\mathrm{ng} / \mathrm{ml})\end{array}$} & \multicolumn{8}{|c|}{ Peak area (serial dilution of positive control material) } & \multirow{2}{*}{$\begin{array}{l}\text { Limit of } \\
\text { detection } \\
(\mathrm{ng} / \mathrm{ml})\end{array}$} \\
\hline & & $\begin{array}{c}\text { No } \\
\text { dilution }\end{array}$ & 2-fold & 4 -fold & 8 -fold & 16-fold & 32 -fold & 64-fold & 128-fold & \\
\hline Benzoylecgonine & 4,000 & 220,565 & 10,9881 & 57,495 & 28,707 & 14,213 & 6,806 & 3,745 & 1,950 & $<31$ \\
\hline Morphine & 3,000 & 5,430 & 3,744 & 2,266 & 1,455 & 940 & 376 & 136 & 48 & $<24$ \\
\hline Nordiazepam & 3,000 & 17,961 & 9,923 & 6,521 & 2,802 & 1,773 & 449 & 390 & 44 & $<24$ \\
\hline Amphetamine & 2,000 & 50,095 & 31,344 & 19,063 & 9,904 & 4,824 & 2,352 & 1,080 & 995 & $<16$ \\
\hline Methamphetamine & 2,000 & 161,765 & 82,626 & 43,463 & 22,618 & 10,641 & 5,240 & 2,695 & 1,456 & $<16$ \\
\hline Nortriptyline & 2,000 & 22,560 & 9,642 & 3,969 & 1,895 & 602 & 83 & ND & ND & 62 \\
\hline Oxazepam & 1,000 & 4,531 & 2,432 & 847 & 521 & 216 & 78 & ND & ND & 31 \\
\hline $\begin{array}{l}\text { 3,4-methylenedioxy- } \\
\text { methamphetamine }\end{array}$ & 750 & 26,949 & 13,746 & 6,291 & 3,630 & 1,566 & 937 & 324 & 234 & $<6$ \\
\hline Methadone & 750 & 52,380 & 22,869 & 9,701 & 4,840 & 2,266 & 787 & 202 & 96 & 6 \\
\hline Propoxyphene & 750 & 48,875 & 21,026 & 10,820 & 5,396 & 2,382 & 956 & 152 & 56 & 6 \\
\hline Phencyclidine & 250 & 1,957 & 952 & 445 & 57 & 84 & ND & ND & ND & 16 \\
\hline Oxycodone & 150 & 902 & 596 & 218 & 101 & ND & ND & ND & ND & 19 \\
\hline
\end{tabular}

ND, not detected.

tamine), methadone and propoxyphene were $<6,6$, and 6 $\mathrm{ng} / \mathrm{ml}$, respectively (Table 2 ).

In total, 473 urine samples from the emergency center were analyzed by UPLC-TMS. A total of 418 patients
$(88.4 \%)$ tested positive for one or more medicinal drugs or drugs of abuse. Twenty-eight drugs were detected at least ten times; the drugs detected most commonly were zolpidem (hypnotics, 21.4\%), ephedrine (sympathomimetic 
Table 3. Distribution of the drugs detected most commonly in the urine of 473 drug-intoxicated patients who visited the emergency center

\begin{tabular}{lc}
\hline \multicolumn{1}{c}{ Drug } & Detected, $\mathrm{n}(\%)$ \\
\hline Zolpidem & $101(21.4)$ \\
Ephedrine & $88(18.6)$ \\
Paracetamol & $86(18.2)$ \\
Chlorpheniramine & $79(16.7)$ \\
Ranitidine & $69(14.6)$ \\
Caffeine & $66(14.0)$ \\
Tramadol & $55(11.6)$ \\
Trazadone & $38(8.0)$ \\
Diphenhydramine & $33(7.0)$ \\
Metoclopramide & $32(6.8)$ \\
Citalopram & $28(5.9)$ \\
Doxylamine & $28(5.9)$ \\
Phenylpropanolamine & $25(5.3)$ \\
Quetiapine & $23(4.9)$ \\
Venlafaxine & $19(4.0)$ \\
Domperidone & $18(3.8)$ \\
7-amino-clonazepam & $17(3.6)$ \\
Atropine & $17(3.6)$ \\
Sulpiride & $17(3.6)$ \\
Topiramate & $16(3.4)$ \\
Fluoxetine & $15(3.2)$ \\
7-amino-flunitrazepam & $14(3.0)$ \\
Atenolol & $14(3.0)$ \\
Propanolol & $14(3.0)$ \\
Nortriptyline & $13(2.7)$ \\
Alprazolam & $12(2.5)$ \\
Ambroxol & $11(2.3)$ \\
\hline
\end{tabular}

amine, 18.6\%), paracetamol (analgesics, 18.2\%) and chlorpheniramine (antihistamine, 16.7\%) (Table 3).

\section{DISCUSSION}

It is clear that clinical laboratories are moving more towards MS-based technology for broad screening of abused drugs and other toxicants. In this study, we established a revised high-throughput UPLC-TMS method for simultaneous screening of drugs most often identified and other toxicants in urine samples detected at the emergency center. This method was validated and was selective, rapid, and robust and showed no compromise of the separation of otherwise interfering peaks.

This method was based on data acquisition associated with targeted MRM screening. ${ }^{14)}$ The MRM method can be compromised either by the number of analytes that can be simultaneously screened, or by the analysis time. However, the acquisition method in this study was arranged into 30 time windows over the chromatographic elution range and could evaluate large panels of analytes within a short time, resulting in improved data efficiency and a number of data points sufficient for peak characteri- zation. The overall cycle time for comprehensive screening was reduced significantly, greatly increasing throughput.

The SSM was used to verify that the method was performing as expected prior to acquiring sample data. The data were obtained by injecting a SSM containing a combination of substances that eluted across the entire chromatographic run.

The LODs showed that each analyte could be measured with acceptable accuracy. The methods had high sensitivity for screening low concentrations of drugs $(6 \mathrm{ng} / \mathrm{ml})$ in urine. The majority of immunochromatographic methods shows much higher cut-off values of $300-1,000 \mathrm{ng} / \mathrm{ml}$. Therefore, UPLC-TMS is highly recommended for detection of low concentrations of drugs in urine.

Additionally, the reproducibility tests using negative and positive QC materials showed results consistent with the target values for all analytes; moreover, these data were in agreement with those reported by the manufacturers.

A total of 473 clinical urine samples from the emergency center were analyzed using UPLC-TMS. Most of the drug intoxicated patients intended to manage uncontrollable pain or commit suicide; therefore, they were admitted to the emergency center with loss of consciousness. A rapid screening of a broad range of drugs is commonly used as the initial work-up to identify the reason for the loss of consciousness. In this study, 418 patients $(88.4 \%)$ tested positive for one or more medicinal drugs or drugs of abuse. Twenty-eight drugs were detected at least 10 times; the drugs detected most commonly were zolpidem, ephedrine, paracetamol, and chlorpheniramine. As seen in other studies, nicotine, caffeine, and lidocaine were commonly encountered during screening for drugs of abuse. Interestingly, theophylline was detected frequently, likely due to it being metabolized by the liver from caffeine.

The major limitation of this method is that the method do not use internal standard and just use the peak area of drug only (not drug and internal standard ratio). Even though the method is qualitative (semi-quantitative) method, sensitivity of mass spectrometry is very vulnerable to matrix effect and the instrument condition. This may cause the errors in the analysis. And the validation of the study was just limited to only 6 drugs for UPLC condition (peak shape and retention time) and only 12 drugs of LOD validation. Therefore most of other drugs (among 177 drugs) were not validated in the study.

Here, the revised and rapid UPLC-TMS method showed excellent performance for the simultaneous screen- 
ing of drugs of abuse in urine samples. Sample preparation was simple and the ability to simultaneously detect a large number of the drugs encountered most commonly significantly enables high throughput, and shortens runtimes. We concluded that this method is a highly specific, reliable and robust technique to screen for a large number of drugs simultaneously and is thus preferred for rapid screening of the substances commonly encountered in emergency cases.

\section{Acknowledgments}

This work was supported by the Soonchunhyang University Research Fund.

\section{REFERENCES}

1. Oiestad EL, Johansen U, Oiestad AM, Christophersen AS. Drug screening of whole blood by ultra-performance liquid chromatography-tandem mass spectrometry. J Anal Toxicol 2011;35:280-293.

2. Lillsunde P, Michelson L, Forsstrom T, Korte T, Schultz E, Ariniemi K, et al. Comprehensive drug screening in blood for detecting abused drugs or drugs potentially hazardous for traffic safety. Forensic Sci Int 1996;77:191-210.

3. Titier K, Castaing N, Le-Déodic M, Le-Bars D, Moore N, Molimard M. Quantification of tricyclic antidepressants and monoamine oxidase inhibitors by high-performance liquid chromatography-tandem mass spectrometry in whole blood. J Anal Toxicol 2007;31:200-207.

4. Sauvage FL, Gaulier JM, Lachâtre G, Marquet P. A fully automated turbulent-flow liquid chromatography-tandem mass spectrometry technique for monitoring antidepressants in human serum. Ther Drug Monit 2006;28:123-130.

5. Wille SM, Maudens KE, Van Peteghem CH, Lambert WE. Development of a solid phase extraction for 13 'new' generation antidepressants and their active metabolites for gas chromatographic-mass spectrometric analysis. $J$ Chromatogr A 2005;1098:19-29.
6. Eichhorst JC, Etter ML, Rousseaux N, Lehotay DC. Drugs of abuse testing by tandem mass spectrometry: a rapid, simple method to replace immunoassays. Clin Biochem 2009; 42:1531-1542.

7. Sadeg N, François G, Petit B, Dutertre-Catella H, Dumontet M. Automated liquid-chromatographic analyzer used for toxicology screening in a general hospital: 12 months' experience. Clin Chem 1997;43:498-504.

8. Adamowicz P, Kała M. Simultaneous screening for and determination of 128 date-rape drugs in urine by gas chromatography-electron ionization-mass spectrometry. Forensic Sci Int 2010;198:39-45.

9. Gunnar T, Mykkänen S, Ariniemi K, Lillsunde P. Validated semiquantitative/quantitative screening of 51 drugs in whole blood as silylated derivatives by gas chromatography-selected ion monitoring mass spectrometry and gas chromatography electron capture detection. J Chromatogr B Analyt Technol Biomed Life Sci 2004;806:205-219.

10. Maurer HH. Advances in analytical toxicology: the current role of liquid chromatography-mass spectrometry in drug quantification in blood and oral fluid. Anal Bioanal Chem 2005;381:110-118.

11. Smink BE, Brandsma JE, Dijkhuizen A, Lusthof KJ, de Gier JJ, Egberts AC, et al. Quantitative analysis of 33 benzodiazepines, metabolites and benzodiazepine-like substances in whole blood by liquid chromatography-(tandem) mass spectrometry. J Chromatogr B Analyt Technol Biomed Life Sci 2004;811:13-20.

12. Maurer HH. Multi-analyte procedures for screening for and quantification of drugs in blood, plasma, or serum by liquid chromatography-single stage or tandem mass spectrometry (LC-MS or LC-MS/MS) relevant to clinical and forensic toxicology. Clin Biochem 2005;38:310-318.

13. Lynch KL, Breaud AR, Vandenberghe $\mathrm{H}, \mathrm{Wu}$ AH, Clarke W. Performance evaluation of three liquid chromatography mass spectrometry methods for broad spectrum drug screening. Clin Chim Acta 2010;411:1474-1481.

14. Rosano TG, Wood M, Swift TA. Postmortem drug screening by non-targeted and targeted ultra-performance liquid chromatography-mass spectrometry technology. J Anal Toxicol 2011;35:411-423. 